\title{
Unit Root of Cumulative Call Rate and Cointegration Analysis of Economic System
}

\author{
Yoji MORITA and Shigeyoshi MIYAGAWA \\ Faculty of Economics, Kyoto Gakuen University \\ Sogabe-cho, Kameoka, Kyoto 621-8555 JAPAN \\ morita-y@kyotogakuen.ac.jp
}

\begin{abstract}
In this paper, a unit root of cumulative call rate is investigated with Monte Carlo experiments. Call rate is shown to be $I(0)$ with a linear trend in $[1970,1998]$. Vector Error Correction model consists of only $I(1)$ variables in a frame work of Johansen's cointegration analysis, and stationary variables like a call rate are often added to the RHS of the resultant VEC model without considering cointegration. However, if cumulative call rate is $I(1)$, this variable should be contained in cointegration analysis. Since call rate has a linear trend, a unit root of cumulative call rate should be checked around a quadratic trend. MacKinnon's critical values for unit root are extended to a quadratic trend case with Monte Carlo simulation experiments. It is shown that cumulative call rate has a unit root with a quadratic trend. Cointegration properties are investigated among cumulative call rate, GDP, money supply and bank loan and VEC model is constructed.
\end{abstract}

\section{Introduction and Problem Statements}

There are a lot of works on unit root and cointegration analysis from empirical and statistical point of view. If a nonstationary variable is contained in the endogeneous and/or exogeneous variables of the system, there is a possibility of spurious regression reported by Granger and Newbold[1]. The importance of stationarity property is emphasized and cointegration analysis is introduced when transforming $I(1)$ variable into $I(0)$ variable by a difference operation.

Let us consider the following example. Let income and money be denoted by $y(t)$ and $m(t)$ respectively. Assume that each variable is $I(1)$ and that $y(t)$ and $m(t)$ are modelled by linear equations:

$$
\begin{aligned}
y(t)= & \mu_{y}+a_{1} y(t-1)+a_{2} y(t-2)+ \\
& +b_{1} m(t-1)+b_{2} m(t-2)+\varepsilon_{y}(t) . \\
m(t)= & \mu_{m}+c_{1} y(t-1)+c_{2} y(t-2)+ \\
& +d_{1} m(t-1)+d_{2} m(t-2)+\varepsilon_{m}(t) .
\end{aligned}
$$

Defining $x(t)=(y(t), m(t))^{\prime}$, Eqs.(1) and (2) are rewritten by a vector form:

$$
x(t)=\mu+\Pi_{1} x(t-1)+\Pi_{2} x(t-2)+\varepsilon(t) .
$$

Using the difference operator $\triangle x(t)=x(t)-x(t-1)$, Eq.(3) can be transformed :

$$
\triangle x(t)=\Gamma \triangle x(t-1)+\Pi x(t-2)+\mu+\varepsilon(t),
$$

where

$$
\Gamma=-\left(I-\Pi_{1}\right)
$$

and where

$$
\Pi=-\left(I-\Pi_{1}-\Pi_{2}\right) .
$$

Since $x(t)$ is nonstationary and $\Pi x(t-2)$ is stationary, the matrix $\Pi$ can be classified by two cases: (case1) $\operatorname{rank} \Pi=0$, i.e., $\Pi$ is the null matrix. (case2) $\operatorname{rank} \Pi=1$, which implies that there are $2 \times 1$ matrices $\alpha$ and $\beta$ such that $\Pi=\alpha \beta^{\prime}$ and that $\beta^{\prime} x(t-2)$ is stationary even though $x(t)$ itself is non-stationary. $\beta$ is called the cointegration vectors. Our interest is concerned with (case2) and Eq.(4) in this case is called VECM (vector error correction model). Johansen[3] gives us the method of rank estimation along with hypothesis test. Hence, if there is no cointegration, an ordinary AR model for $(\triangle y(t), \triangle m(t))$ is available, while, in the existence of cointegration, error correction terms should be added to the above AR model. Now, let us consider another situation such that a nonstationary process $y(t)$ is combined with a stationary process $r(t)$. Johansen's method is not directly applicable to this case since all the variables should be nonstationary in his frame work. Therefore many papers deal this case in such a manner that a set of sataionary variables $(\triangle y(t), r(t))$ is used to construct AR model. However, letting $R(t) \equiv r(1)+r(2)+\cdots+r(t)$, that is, $\triangle R(t) \equiv r(t)$, and assuming that $\mathrm{R}(\mathrm{t})$ is nonstationary, AR model applied to $(\triangle y(t), \triangle R(t))$ is not correct if we do not investigate a cointegration property between $y(t)$ and $R(t)$. 


\section{The Empirical Analysis}

[ Data Description ] The data employed here is Japanese data, quarterly observed from 1970:1 through 1998:4 and original(i.e.,seasonally unadjusted). They are obtained from Nikkei Needs Database. Denoting the GDP deflator by $p(t)$, main variables used here are real GDP,real money,real loan,call rate and cumulative call rate to be listed as:

$$
\begin{aligned}
y(t) & =\ln (\text { real } G D P(t)) \\
m(t) & =\ln \left(M_{2}+C D / p(t)\right) \\
l(t) & =\ln (\text { loan } / p(t)) \\
r(t) & =\text { call rate }(t) \\
R(t) & =\sum_{i=1}^{t} r(i) .
\end{aligned}
$$

[Unit Root Test] Dicky-Fuller test is applied for check of stationarity. This test requires to calculate t-ratio of the parameter $\lambda_{0}$ in the following equation:

$x(t)-x(t-1)=a+b t+\lambda_{0} x(t-1)+\sum_{i=1}^{k_{0}} \lambda_{i} \triangle x(t-i)+\Psi D(t)$,

where $x(t)$ is a scalar variable to be tested, the delay parameter $k_{0}$ is decided by $A I C$ criterion and where $D(t)$ is a seasonal dummy variable with elements $D_{2}(t)$, $D_{3}(t)$ and $D_{4}(t)$. If the null hypothesis $\lambda_{0}=0$ is rejected, then the $x(t)$ process does not have a unit root and becomes stationary. Table 1 shows results of the test with $\operatorname{trend}(b \neq 0)$ and without trend $(b=0)$.

Table 1 Unit Root Tests

\begin{tabular}{|c|c|c|c|c|}
\hline var. & lags & with trend & lags & without trend \\
\hline $\mathrm{y}$ & 5 & -1.05187 & 5 & -2.18168 \\
$\mathrm{~m}$ & 2 & -2.90397 & 2 & -2.01160 \\
$\mathrm{l}$ & 1 & -0.91695 & 1 & -1.82782 \\
$\mathrm{r}$ & 2 & $-4.06200 * * *$ & 2 & $-2.63382 *$ \\
\hline
\end{tabular}

where $* * *, * *$ and $*$ denote respectively rejection of the hypothesis at $1 \%, 5 \%$ and $10 \%$ significance level. Critical values tabulated by MacKinnon's table[2] are $-4.04,-3.45$ and -3.15 for $1 \%, 5 \%$ and $10 \%$ in with trend, while the values are $-3.49,-2.89$ and -2.58 for $1 \%$, $5 \%$ and $10 \%$ significance levels respectively without trend. The lag order is decided by AIC. We conclude that $y(t), m(t)$ and $l(t)$ are nonstationary and $r(t)$ is stationary with trend. Cumulative call rate $R(t)$ is somewhat difficult to judge the unit root. Since $\Delta r(t)$ has a drift, that is, $E\{\triangle r(t)\}=-0.0713$, the unit root test of $r(t)$ should be done around a linear time trend. The result of ADF test implies that the null hypothesis

\begin{tabular}{|c|c|}
\hline \multicolumn{2}{|c|}{$\begin{array}{l}\text { Series: D(CALL) } \\
\text { Sample 1970:2 1998:4 } \\
\text { Observations } 115\end{array}$} \\
\hline Mean & -0.071304 \\
\hline Median & -0.053333 \\
\hline Maximum & 3.266667 \\
\hline Minimum & -2.323333 \\
\hline Std. Dev. & 0.779814 \\
\hline Skewness & 0.791532 \\
\hline Kurtosis & 6.766423 \\
\hline Jarque-Bera & 79.98266 \\
\hline Probability & 0.000000 \\
\hline
\end{tabular}
of unit root is rejected with $1 \%$ significance level.

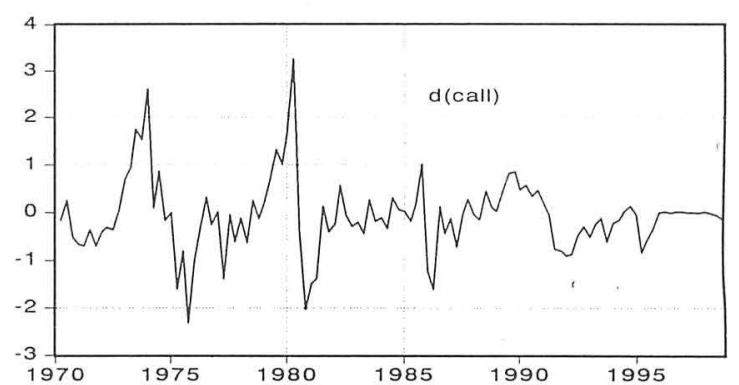

Figure 1: behavior of $\triangle r(t)$

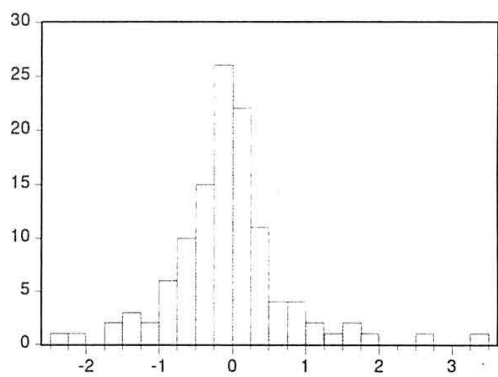

Figure 2: histogram and statistics of $\triangle r(t)$

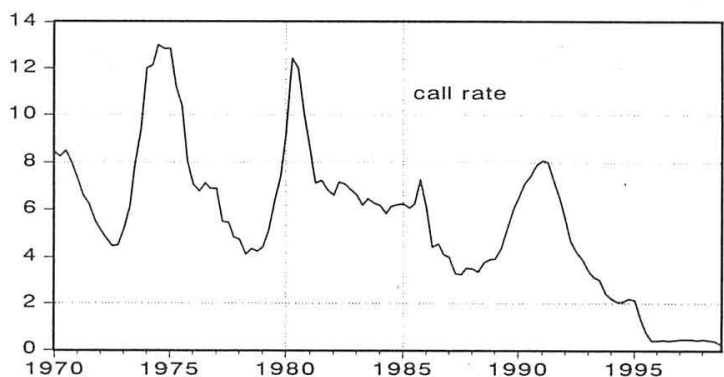

Figure 3: behavior of call rate $r(t)$

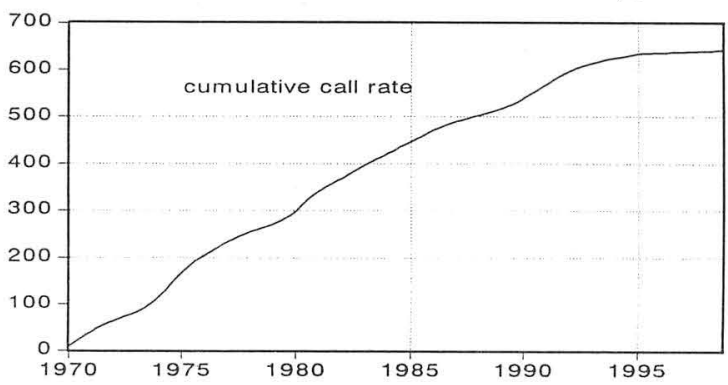

Figure 4: behavior of cumulative call rate $R(t)$ 
In the next step, the unit root of cumulative call rate $R(t)$ should be examined. Since $\triangle R(t)=r(t)$ has a linear trend, $R(t)$ itself may fluctuate around a quadratic trend.

Letting $L$ be a lagged operator, we consider the $z(t)$ -process with a quadratic trend.

$$
\left(1-\phi_{1} L\right)(1-\rho L)\left\{z(t)-\left(\mu+\beta_{1} t+\beta_{2} t^{2}\right)\right\}=u(t)
$$

where $u(t)$ is a series of $i . i . d . N(0,1)$ variables. The L.H.S. of the above equation becomes

$$
\begin{aligned}
\text { L.H.S. }= & z(t)-\left(\phi_{1}+\rho\right) z(t-1)+\phi_{1} \rho z(t-2) \\
& -\mu^{\prime}-\beta_{1}^{\prime} t-\beta_{2}^{\prime} t^{2} \\
= & \triangle z(t)+\left(1-\phi_{1}\right)(1-\rho) z(t-1) \\
& -\phi_{1} \rho \Delta z(t-1)-\mu^{\prime}-\beta_{1}^{\prime} t-\beta_{2}^{\prime} t^{2},(1
\end{aligned}
$$

where

$$
\begin{aligned}
\mu^{\prime}= & \left(1-\phi_{1}\right)(1-\rho) \mu+\left\{\left(\phi_{1}+\rho\right)-2 \phi_{1} \rho\right\} \beta_{1} \\
& +\left\{\left(\phi_{1}+\rho\right)-4 \phi_{1} \rho\right\} \beta_{2},
\end{aligned}
$$

$$
\beta_{1}^{\prime}=\left(1-\phi_{1}\right)(1-\rho) \beta_{1}+2\left\{\left(\phi_{1}+\rho\right)-2 \phi_{1} \rho\right\} \beta_{2},
$$

$$
\beta_{2}^{\prime}=\left(1-\phi_{1}\right)(1-\rho) \beta_{2} .
$$

Finally, Eq.(13) is rewritten by

$$
\triangle z(t)=\mu^{\prime}+\beta_{1}^{\prime} t+\beta_{2}^{\prime} t^{2}+\alpha_{0} z(t-1)+\alpha_{1} \Delta z(t-1)+u(t),
$$

where

$$
\alpha_{0}=-\left(1-\phi_{1}\right)(1-\rho) \text {, and } \alpha_{1}=\phi_{1} \rho .
$$

Inserting the relation $\rho=1$ into Eq.(18), the null hypothesis of unit root is stated with $\beta_{2}^{\prime}=0$ and $\alpha_{0}=0$

$$
\triangle z(t)=\mu^{\prime}+\beta_{1}^{\prime} t+\phi_{1} \triangle z(t-1)+u(t),
$$

where

$$
\begin{aligned}
& \mu^{\prime}=\left(1-\phi_{1}\right) \beta_{1}+\left(1-3 \phi_{1}\right) \beta_{2}, \\
& \beta_{1}^{\prime}=2\left(1-\phi_{1}\right) \beta_{2} .
\end{aligned}
$$

The alternative hypothesis is given by Eq.(18) with $\beta_{2}^{\prime} \neq 0$ and $\alpha_{0} \neq 0$. The unit root should be judged by comparing t-statistics of the estimated $\alpha_{0}$ with critical values. These critical values are given by Monte Carlo experiments.

[Monte Carlo Experiments] Calculation of critical values for unit root is carried out.

(1)step-1: Eq.(20) in the null hypothesis is simulated with the sample size $T=113$.

(2)step-2: Based on the data given in step-1, the parameter $\alpha_{0}$ in Eq.(18) and its t-statistics are calculated. (3)step-3: Each simulation experiment in step-1 and step-2 consists of 25,000 replications for a single value of $\mathrm{T}$ which is determined by the sample size of data period 1970:1 to 1998:4.

(4)step-4: The 25,000 values of t-statistics of estimated $\alpha_{0}$ are shown in the cumulative distribution function(denoted by cdf).

Figures 5 and 6 depict the cdf and its expanded map respectively. The cumulative call rate $R(t)$ in the period 1970:1 to 1998:4 is identified in Eq.(18) and tstatistics of $\alpha_{0}$ becomes to be -3.38 . This value gives us the p-value 0.14 , which can not reject the null hypothesis of unit root with $10 \%$ significance level.

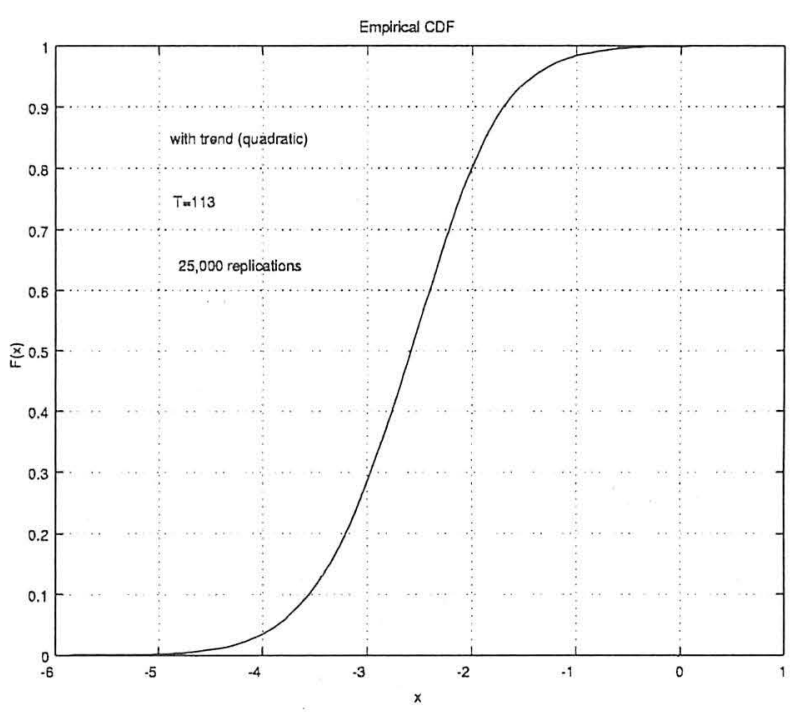

Figure 5: Empirical CDF of t-statistics of estimated $\alpha_{0}$

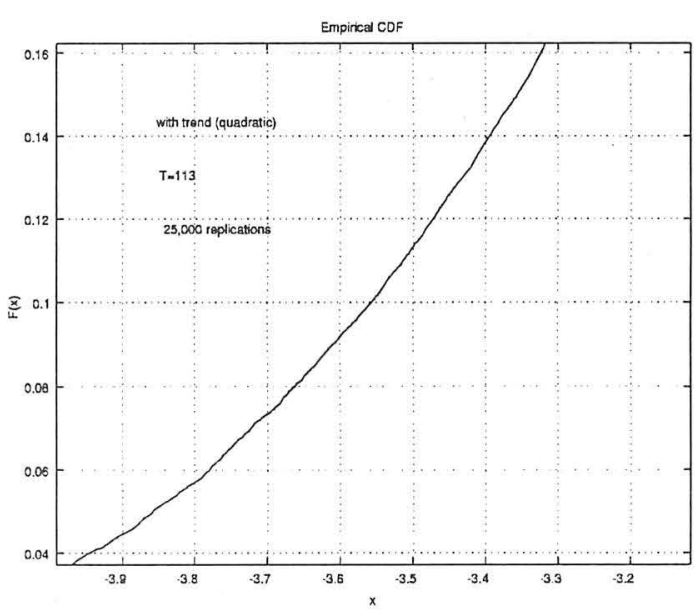

Figure 6: Expanded map of Empirical CDF

[ Cointegration Property(Johansen Test[3]) ] Johansen gives us the method of rank estimation along with hypothesis test. Table 2 is results of Johansen test. 
Table 2 Cointegration Tests (Johansen) on $(y, m, l, R)$

\begin{tabular}{|c|c|c|c|c|}
\hline $\begin{array}{c}\text { Eigen } \\
\text { values }\end{array}$ & L.Ratio & $\begin{array}{c}\text { critical } \\
(5 \%)\end{array}$ & $\begin{array}{c}\text { critical } \\
(1 \%)\end{array}$ & $\begin{array}{c}\text { hypothesized } \\
\text { No. of } C E\end{array}$ \\
\hline 0.34 & 74.76 & 54.64 & 61.24 & $r=0 * *$ \\
0.16 & 28.12 & 34.55 & 40.49 & $r \leq 1$ \\
0.058 & 8.85 & 18.17 & 23.46 & $r \leq 2$ \\
0.019 & 2.17 & 3.74 & 6.4 & $r \leq 3$ \\
\hline
\end{tabular}

where $* *(*)$ denotes rejection of hypothesis at $1 \%$ $(5 \%)$ significance level and where lagged differences are $n=4$ by AIC.

From Johansen test, $x(t)=(y(t), m(t), l(t), R(t))$ has a cointegrating vector, that is, $r=1$. The cointegrating vector is calculated as:

$$
\begin{aligned}
y(t)= & c_{1}+c_{2} t+c_{3} m(t)+c_{4} l(t)+c_{5} R(t) \\
= & -3.0266-0.01697 t \\
& +2.4596 m(t)-1.0539 l(t)+0.00156 R(t), \\
& \quad(1.31074) \quad(0.65907) \quad(0.00076)(23)
\end{aligned}
$$

where $t$ means time trend, the number in parentheses are the asymptotic standard errors and where Eq.(23) contains seasonal dummy variables on the RHS of their equations.

The error correcting term $z_{1}(t)$ can be defined as the residual of the above relation and is described by

$$
z_{1}(t)=y(t)-c_{1}-c_{2} t-c_{3} m(t)-c_{4} l(t)-c_{5} R(t),
$$

In order to support this result, Johansen tests are carried out in case of several combinations of variables to be shown in Table 3. See Appendix.

[ Basic System Model] Our aim is to research how monetary policy (call rate $r(t)$ ) transmits to output $\triangle y(t)$ through channels of $\Delta m(t), \Delta l(t)$ and $z_{1}(t)$. Since cointegration in Johansen test assumes a set of variables with $I(1)$ and call rate is, on the contrary, stationary in its level (i.e., $I(0)$ ), an introduction of $I(1)$ variable $R(t)$ plays an important role to the analysis of monetary transmission mechanism. The VEC model is described by

$$
\begin{aligned}
\triangle y(t)= & \sum_{i=1}^{k} a_{1 i} \triangle y(t-i)+\sum_{i=1}^{k} a_{2 i} \triangle m(t-i) \\
& +\sum_{i=1}^{k} a_{3 i} \triangle l(t-i)+\sum_{i=1}^{k} a_{4 i} \triangle R(t-i) \\
& +a_{5} z_{1}(t-1)+\mu_{y}+a_{6} t \\
& +\sum_{j=2}^{4} s_{y j} D_{j}(t)+\sigma_{y} \varepsilon_{y}(t)
\end{aligned}
$$

$$
\begin{aligned}
\triangle m(t)= & \sum_{i=1}^{k} b_{1 i} \triangle y(t-i)+\sum_{i=1}^{k} b_{2 i} \triangle m(t-i) \\
& +\sum_{i=1}^{k} b_{3 i} \triangle l(t-i)+\sum_{i=1}^{k} b_{4 i} \triangle R(t-i) \\
& +b_{5} z_{1}(t-1)+\mu_{m}+b_{6} t \\
& +\sum_{j=2}^{4} s_{m j} D_{j}(t)+\sigma_{m} \varepsilon_{m}(t)
\end{aligned}
$$

$$
\begin{aligned}
\Delta l(t)= & \sum_{i=1}^{k} c_{1 i} \triangle y(t-i)+\sum_{i=1}^{k} c_{2 i} \triangle m(t-i) \\
& +\sum_{i=1}^{k} c_{3 i} \triangle l(t-i)+\sum_{i=1}^{k} c_{4 i} \triangle R(t-i) \\
& +c_{5} z_{1}(t-1)+\mu_{l}+c_{6} t \\
& +\sum_{j=2}^{4} s_{l j} D_{j}(t)+\sigma_{l} \varepsilon_{l}(t)
\end{aligned}
$$

$\triangle R(t)$

$$
\begin{aligned}
= & \sum_{i=1}^{k} d_{1 i} \triangle y(t-i)+\sum_{i=1}^{k} d_{2 i} \triangle m(t-i) \\
& +\sum_{i=1}^{k} d_{3 i} \triangle l(t-i)+\sum_{i=1}^{k} d_{4 i} \triangle R(t-i) \\
& +d_{5} z_{1}(t-1)+\mu_{\text {call }}+d_{6} t \\
& +\sum_{j=2}^{4} s_{c j} D_{j}(t)+\sigma_{\text {call }} \varepsilon_{\text {call }}(t)
\end{aligned}
$$

where $\varepsilon_{y}(t), \cdots, \varepsilon_{\text {call }}(t)$ are sequences of i.i.d. random variables with standard deviations $\sigma_{y}, \cdots, \sigma_{\text {call }}$ respectively and where $t$ denotes a time trend. From the relation $\triangle R(t) \equiv r(t)$, it should be noted that the model constructed above represents an economic system driven by call rate. The delay order $k$ was decided by $A I C$ to be $k=4$. Basic behaviors of $\triangle y(t), \triangle m(t)$, $\triangle l(t)$ and $r(t)$ are depicted in Fig.7 in a form of impulse responses subjected to the shock of $r(t)$.

The monetary transmission problem can be discussed by the basic model derived above, see Morita et al.[4].

\section{Conclusions}

A new variable $R(t)$ which is cumulative call rate was introduced and an economic system model driven by 

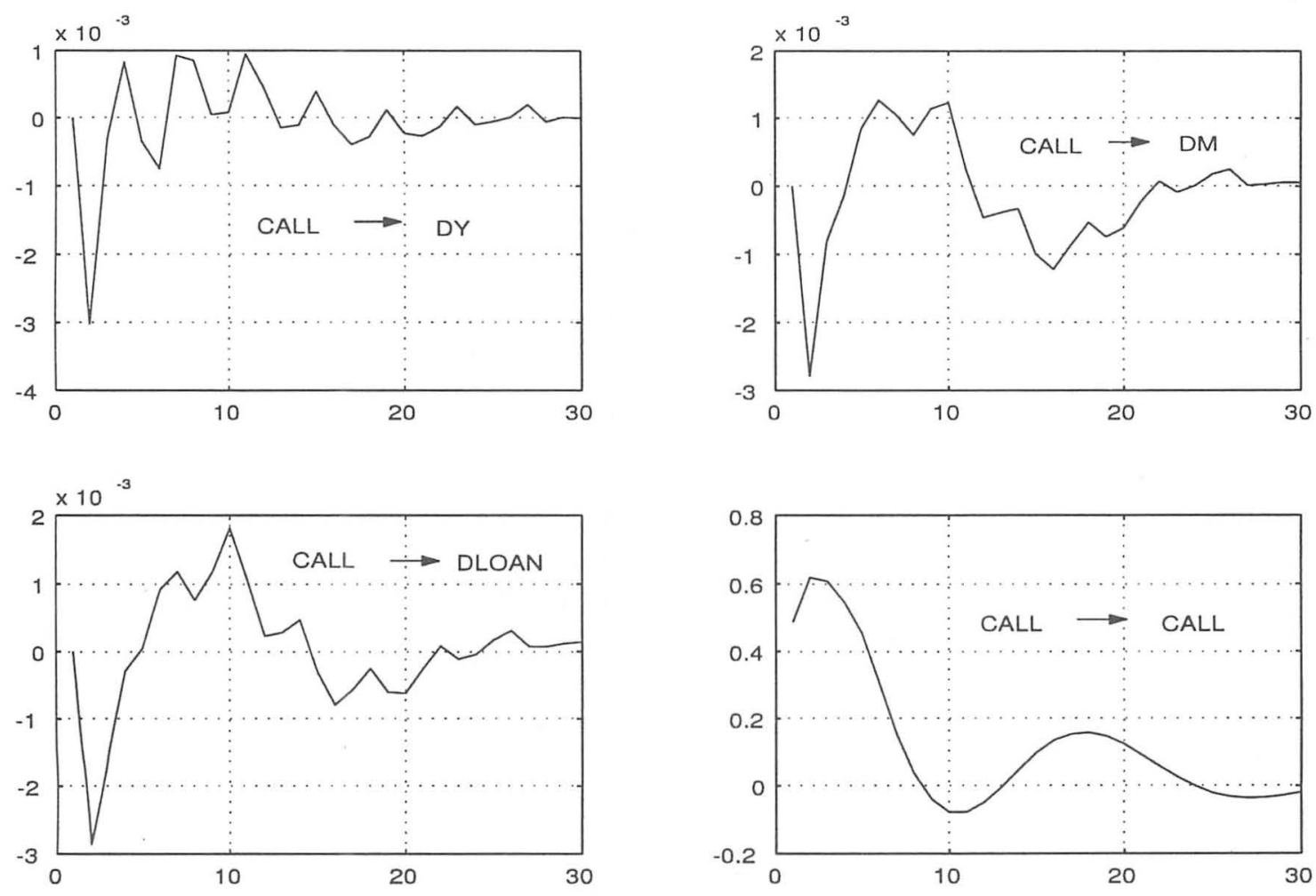

Figure 7: Impulse Responses in Basic System Model

call rate was reasonablly constructed associated with cointegration analysis. A combination of nonstationary variables and a stationary variable(call rate) was theoretically analyzed within Johansen's cointegration frame work. The VEC model was calculated and the resultant impulse responses were shown.

\section{References}

[1] Granger, C.W.J. and Newbold, P. Forecasting Economic Time Series, (2nd ed.),Academic Press,1986.

[2] Mackinnon, J.G. Critical Values for Cointegration Tests, in R.F.Engle and C.W.J.Granger ed., Long-Run Economic Relationships:Oxford University Press,1991, pp.267-276.

[3] Johansen, S. Estimation and Hypothesis Testing of Cointegration Vectors in Gaussian Vector Autoregressive Models, Econometrica, Vol.59, 1991, pp.1551-1580.

[4] Morita, Y., Miyagawa, S. and Nakazaki, H. On the Role of Call Rate in Cointegrating Analysis of Monetary Transmission Mechanism, The Kyotogakuen University Review, March, 2001, pp.41-54. 
Appendix Table 3 Summary of Cointegration Tests (Johansen)

\begin{tabular}{|l|l|l|l|}
\hline variables & $n=2$ & $n=3$ \\
\hline$(y, m, l, R)$ & $r=0 * * \quad r \leq 1 \quad r \leq 2 \quad r \leq 3 *$ & $r=0 * * \quad r \leq 1 \quad r \leq 2 \quad r \leq 3$ \\
$(y, m, R)$ & $r=0 * \quad r \leq 1 \quad r \leq 2$ & $r=0 * * \quad r \leq 1 \quad r \leq 2$ \\
$(y, l, R)$ & $r=0 * * \quad r \leq 1 * \quad r \leq 2 *$ & $r=0 * * \quad r \leq 1 \quad r \leq 2 *$ \\
\hline$(y, R)$ & $r=0 * * \quad r \leq 1 *$ & $r=0 * * \quad r \leq 1 *$ \\
$(m, R)$ & $r=0 \quad r \leq 1$ & $r=0 * \quad r \leq 1$ \\
$(l, R)$ & $r=0 \quad r \leq 1 *$ & $r=0 * \quad r \leq 1 *$ \\
\hline$(y, m)$ & $r=0 \quad r \leq 1 *$ & $r=0 * * \quad r \leq 1$ \\
$(y, l)$ & $r=0 \quad r \leq 1$ & $r=0 \quad r \leq 1$ \\
$(y, m, l)$ & $r=0 \quad r \leq 1 \quad r \leq 2$ & $r=0 * \quad r \leq 1 \quad r \leq 2$ \\
\hline
\end{tabular}

\begin{tabular}{|l|l|l|l|}
\hline variables & $n=4$ & $n=5$ \\
\hline$(y, m, l, R)$ & $r=0 * * \quad r \leq 1 \quad r \leq 2 \quad r \leq 3$ & $r=0 * \quad r \leq 1 \quad r \leq 2 \quad r \leq 3$ \\
$(y, m, R)$ & $r=0 \quad r \leq 1 \quad r \leq 2$ & $r=0 \quad r \leq 1 \quad r \leq 2$ \\
$(y, l, R)$ & $r=0 * \quad r \leq 1 \quad r \leq 2 *$ & $r=0 * \quad r \leq 1 \quad r \leq 2 *$ \\
\hline$(y, R)$ & $r=0 * \quad r \leq 1 *$ & $r=0 * \quad r \leq 1 *$ \\
$(m, R)$ & $r=0 * \quad r \leq 1$ & $r=0 \quad r \leq 1$ \\
$(l, R)$ & $r=0 \quad r \leq 1 *$ & $r=0 \quad r \leq 1 *$ \\
\hline$(y, m)$ & $r=0 \quad r \leq 1$ & $r=0 \quad r \leq 1$ \\
$(y, l)$ & $r=0 \quad r \leq 1 \quad r=0 \quad r \leq 1$ \\
$(y, m, l)$ & $r=0 \quad r \leq 1 \quad r \leq 2$ & $r=0 \quad r \leq 1 \quad r \leq 2$ \\
\hline
\end{tabular}

\begin{tabular}{|l|l|l|l|}
\hline variables & $n=6$ & $n=7$ \\
\hline$(y, m, l, R)$ & $r=0 * \quad r \leq 1 \quad r \leq 2 \quad r \leq 3$ & $r=0 * * \quad r \leq 1 \quad r \leq 2 \quad r \leq 3$ \\
$(y, m, R)$ & $r=0 \quad r \leq 1 \quad r \leq 2$ & $r=0 \quad r \leq 1 \quad r \leq 2 *$ \\
$(y, l, R)$ & $r=0 \quad r \leq 1 \quad r \leq 2 *$ & $r=0 * \quad r \leq 1 \quad r \leq 2 * *$ \\
\hline$(y, R)$ & $r=0 \quad r \leq 1 * *$ & $r=0 \quad r \leq 1 *$ \\
$(m, R)$ & $r=0 \quad r \leq 1$ & $r=0 \quad r \leq 1 *$ \\
$(l, R)$ & $r=0$ & $r \leq 1 *$ & $r=0 \quad r \leq 1 *$ \\
\hline$(y, m)$ & $r=0$ & $r \leq 1$ & $r=0 \quad r \leq 1$ \\
$(y, l)$ & $r=0$ & $r \leq 1$ & $r=0 * \quad r \leq 1$ \\
$(y, m, l)$ & $r=0$ & $r \leq 1 \quad r \leq 2$ & $r=0 \quad r \leq 1 \quad r \leq 2$ \\
\hline
\end{tabular}

\begin{tabular}{|l|l|l|l|}
\hline variables & $n=8$ & $n=9$ \\
\hline$(y, m, l, R)$ & $r=0 * * \quad r \leq 1 * \quad r \leq 2 \quad r \leq 3$ & $r=0 * * \quad r \leq 1 * \quad r \leq 2 \quad r \leq 3$ \\
$(y, m, R)$ & $r=0 \quad r \leq 1 \quad r \leq 2 * *$ & $r=0 \quad r \leq 1 * \quad r \leq 2 *$ \\
$(y, l, R)$ & $r=0 * \quad r \leq 1 \quad r \leq 2 *$ & $r=0 * * \quad r \leq 1 \quad r \leq 2 *$ \\
\hline$(y, R)$ & $r=0 \quad r \leq 1 *$ & $r=0 * \quad r \leq 1 * *$ \\
$(m, R)$ & $r=0 \quad r \leq 1 * *$ & $r=0 * \quad r \leq 1 * *$ \\
$(l, R)$ & $r=0 \quad r \leq 1 *$ & $r=0 \quad r \leq 1 *$ \\
\hline$(y, m)$ & $r=0 \quad r \leq 1$ & $r=0 \quad r \leq 1$ \\
$(y, l)$ & $r=0 * \quad r \leq 1 *$ & $r=0 * \quad r \leq 1 *$ \\
$(y, m, l)$ & $r=0 \quad r \leq 1 \quad r \leq 2$ & $r=0 \quad r \leq 1 \quad r \leq 2$ \\
\hline
\end{tabular}

